\title{
Allele frequencies for 27 Y-STR loci with U.S. Caucasian, African American, and Hispanic samples
}

\author{
John M. Butler*, Amy E. Decker, Peter M. Vallone, Margaret C. Kline \\ Biotechnology Division, National Institute of Standards and Technology, Gaithersburg, MD 20899-8311, USA
}

Received 26 January 2005; received in revised form 22 February 2005; accepted 22 February 2005

Available online 30 March 2005

\begin{abstract}
A total of 263 U.S. Caucasians, 260 African Americans and 140 U.S. Hispanics or a subset of 31 Caucasians, 32 African Americans, and 32 Hispanics were typed for 27 Y-chromosome short tandem repeat (Y-STR) markers: DYS444, DYS446, DYS449, DYS463, DYS485, DYS490, DYS495, DYS504, DYS505, DYS508, DYS520, DYS522, DYS525, DYS532, DYS533, DYS534, DYS540, DYS556, DYS557, DYS570, DYS575, DYS576, DYS594, DYS632, DYS635, DYS641, and DYS643. Allele frequencies for each locus are reported along with nomenclature based on sequence analysis.

(C) 2005 Elsevier Ireland Ltd. All rights reserved.

Keywords: Short tandem repeat; DNA; Y-chromosome; Y-STR; Population data; DYS444; DYS446; DYS449; DYS463; DYS485; DYS490; DYS495; DYS504; DYS505; DYS508; DYS520; DYS522; DYS525; DYS532; DYS533; DYS534; DYS540; DYS556; DYS557; DYS570; DYS575; DYS576; DYS594; DYS632; DYS635; DYS641; DYS643; Y-GATA-C4; U.S. Caucasian; African American; U.S. Hispanic
\end{abstract}

Population samples: Anonymous liquid blood samples with self-identified ethnicities were purchased from Interstate Blood Bank, Inc. (Memphis, TN) and Millennium Biotech, Inc. (Ft. Lauderdale, FL). All samples were previously examined with 15 autosomal short tandem repeats and the amelogenin sex-typing marker using the AmpFISTR Identifiler kit (Applied Biosystems, Foster City, CA) to verify that each sample was unique [1].

N: Two hundred and sixty U.S. Caucasians, 260 African Americans, and 140 U.S. Hispanics were typed for 27 Ychromosome short tandem repeat (Y-STR) markers. For six of the loci only a subset of each population group was examined that included 31 Caucasians, 32 African Americans, and 32 Hispanics.

DNA extraction: Blood samples were extracted using a modified salting out procedure [2] as described previously [1].

\footnotetext{
* Corresponding author. Tel.: +1 301975 4049; fax: +1 3019758505 .

E-mail address: john.butler@nist.gov (J.M. Butler).
}

Quantification: Extracted DNA was quantified using UV spectrophotometry followed by a PicoGreen assay [3] to adjust concentrations to approximately $1 \mathrm{ng} / \mu \mathrm{L}$ (see [1]).

Y-STR markers: Table 1 lists information on the 27 Y-STR markers examined as part of this study (see also http://www.cstl.nist.gov/biotech/strbase/y_strs.htm). The PCR primer sequences used in this study were those present in the Genome Database (GDB; http://www.gdb.org). In an attempt to generate smaller PCR products for some of the Y-STR loci, new primers were designed for DYS485 (-129 bp), DYS490 ( $-38 \mathrm{bp}$ ), and DYS495 ( $-77 \mathrm{bp}$ ) with the size reduction relative to the GDB generated amplicons shown in parentheses. Fluorescent dyes 6-FAM, VIC, or NED were included on the $5^{\prime}$-end of the forward primers to create blue, green, or yellow-labeled PCR products. The reverse primers included an extra seven base tail (shown in lower case letters) to promote non-template addition. The DYS635 (Y-GATA-C4) data were obtained as part of an evaluation of a beta-test version of the Yfiler kit (Applied Biosystems). The exact chromosomal locations were ascertained using 
Table 1

Information on the 27 Y-STR loci examined in this study

\begin{tabular}{|c|c|c|c|c|}
\hline $\begin{array}{l}\text { Y-STR } \\
\text { locus }\end{array}$ & Primer sequence $\left(5^{\prime} \rightarrow 3^{\prime}\right)$ & $\begin{array}{l}\text { GenBank accession } \\
\text { (repeat position) }\end{array}$ & $\begin{array}{l}\text { GenBank reference } \\
\text { Allele }\end{array}$ & $\begin{array}{l}\text { Y-chromosome } \\
\text { position }(\mathrm{Mb})\end{array}$ \\
\hline DYS444 & $\begin{array}{l}\text { NED-TCTAAGGGATCCAAAGGCAGAA } \\
\text { gtttcttGTGTGAACCATTTGGCATGTTTA }\end{array}$ & AC007043 (nt75953...76008) & (TAGA)14 & 17.664 \\
\hline DYS446 & $\begin{array}{l}\text { FAM-TATTTTCAGTCTTGTCCTGTC } \\
\text { gtttcttAAATGTATGGCCAACATAGCAAAACCA }\end{array}$ & AC006152 (nt21421...21490) & (TCTCT)14 & 3.175 \\
\hline DYS449 & $\begin{array}{l}\text { NED-CCTGGAAGTGGAGTTTGCTGT } \\
\text { gtttcttTGGAGTCTCTCAAGCCTGTTCTA }\end{array}$ & AC051663 (nt93299. . .93464) & $\begin{array}{l}(\text { TTTC) } 15 \ldots \\
(\text { TTTC } 14=\mathbf{2 9}\end{array}$ & 8.261 \\
\hline DYS463 & $\begin{array}{l}\text { FAM-AATTCTAGGTTTGAGCAAAGACA } \\
\text { gtttcttATGAGGTTGTGTGACTTGACTG }\end{array}$ & AC007275 (nt64620...64739) & $\begin{array}{l}(\mathrm{AAAGG})_{7}(\mathrm{AAGGG})_{15} \\
(\mathrm{AAGGA})_{2}=\mathbf{2 4}\end{array}$ & 7.687 \\
\hline DYS485 & $\begin{array}{l}\text { NED-CCTGGGTGACAAGAGTTATACTCT } \\
\text { gtttcttGCAGACTTCGCCACTACATAAT }\end{array}$ & AC009233 (nt86438...86485) & (TTA)16 & 20.488 \\
\hline DYS490 & $\begin{array}{l}\text { VIC-CTGAGCTGAGATCACGCC } \\
\text { gtttcttACGATATGAAAAAGCAGTATGTCCT }\end{array}$ & AC019058 (nt32127. . .32162) & (TTA)12 & 3.487 \\
\hline DYS495 & $\begin{array}{l}\text { FAM-AGCAAACTTTGAAGCCAGAAAG } \\
\text { gtttcttCTTGGGCAACAGAGCGAGA }\end{array}$ & AC004474 (nt49600. . .49644) & (AAT)15 & 13.449 \\
\hline DYS504 & $\begin{array}{l}\text { FAM-TCTACACCACTGTGCCAAGC } \\
\text { gtttcttGGCAACAGAGCAACCCTCT }\end{array}$ & AC006157 (nt11761...117832) & $($ TCCT) 18 & 2.946 \\
\hline DYS505 & $\begin{array}{l}\text { FAM-TCTGGCGAAGTAACCCAAAC } \\
\text { gtttcttTCGAGTCAGTTCACCAGAAGG }\end{array}$ & AC012078 (nt50281...50328) & $($ TCCT) 12 & 3.684 \\
\hline DYS508 & $\begin{array}{l}\text { VIC-ACAATGGCAATCCCAAATTC } \\
\text { gtttcttGAACAAATAAGGTGGGATGGAT }\end{array}$ & AC006462 (nt29187. . .29230) & (TATC)11 & 16.232 \\
\hline DYS520 & $\begin{array}{l}\text { NED-AACAGCCTGCCCAACATAGT } \\
\text { gtttcttACCATCATGCCCTGCAATA }\end{array}$ & AC007275 (nt151532...151622) & $\begin{array}{l}(\mathrm{ATAG})_{10} \\
(\mathrm{ATAC})_{10}=\mathbf{2 0}\end{array}$ & 7.774 \\
\hline DYS522 & $\begin{array}{l}\text { VIC-CCTTTGAAATCATTCATAATGC } \\
\text { gtttcttTCATAAACAGAGGGTTCTGG }\end{array}$ & AC007247 (nt70433...70472) & (GATA)10 & 7.459 \\
\hline DYS525 & $\begin{array}{l}\text { VIC-ATTCACACCATTGCACTCCA } \\
\text { gtttcttCCATCTGTTTATCTTCCCATCA }\end{array}$ & AC010104 (nt92809. . .92848) & (TAGA)10 & 7.120 \\
\hline DYS532 & $\begin{array}{l}\text { NED-TTGGTTTTATGCCTTTCACT } \\
\text { gtttcttTAGGTGACAGAGCAGGATTC }\end{array}$ & AC016991 (nt68764...68819) & $($ CTTT) 14 & 8.423 \\
\hline DYS533 & $\begin{array}{l}\text { VIC-CATCTAACATCTTTGTCATCTACC } \\
\text { gtttcttTGATCAGTTCTTAACTCAACCA }\end{array}$ & AC053516 (nt2747...2794) & $(\mathbf{A T C T}) 12$ & 16.831 \\
\hline DYS534 & $\begin{array}{l}\text { NED-CATCTACCCAACATCCATCTA } \\
\text { gtttcttGACAAAGATGTTAGATGAATAGACA }\end{array}$ & AC053516 (nt2497...2556) & (CTTT) 15 & 16.831 \\
\hline DYS540 & $\begin{array}{l}\text { NED-GACCGTGTACTCTGGCCAAT } \\
\text { gtttcttCAGGAGGCTAGCTCAGGAGA }\end{array}$ & AC010135 (nt147642...147689) & (TTAT)12 & 17.003 \\
\hline DYS556 & $\begin{array}{l}\text { FAM-TGCTGTCACATCACCAATGA } \\
\text { gtttcttTTTGGTTGCTGAAGCATTGA }\end{array}$ & AC011745 (nt19776...19819) & (AATA)11 & 20.940 \\
\hline DYS557 & $\begin{array}{l}\text { VIC-TTTTCTGTGCCAAGCCTACA } \\
\text { gtttcttTCTAATGCACCTTGAGGGATG }\end{array}$ & AC007876 (nt86525 . .86592) & (TTTC)16 & 21.573 \\
\hline DYS570 & $\begin{array}{l}\text { NED-GAACTGTCTACAATGGCTCACG } \\
\text { gtttcttTCAGCATAGTCAAGAAACCAGACA }\end{array}$ & AC012068 (nt43502. . .43569) & (TTTC)17 & 6.905 \\
\hline DYS575 & $\begin{array}{l}\text { FAM-GGTGGTGGACATCCGTAATC } \\
\text { gtttcttAGTAATGGGATGCTGGGTCA }\end{array}$ & AC007247 (nt91065. . .91104) & $(\mathbf{A A A T}) \mathbf{1 0}$ & 7.479 \\
\hline DYS576 & $\begin{array}{l}\text { FAM-TTGGGCTGAGGAGTTCAATC } \\
\text { gtttcttGGCAGTCTCATTTCCTGGAG }\end{array}$ & AC010104 (nt69314...69381) & $(\mathbf{A A A G}) 17$ & 7.096 \\
\hline DYS594 & $\begin{array}{l}\text { VIC-GATGTGCCTAATGCCACAGA } \\
\text { gtttcttCCCTGGTGTTAATCGTGTCC }\end{array}$ & AC010137 (nt50161...50210) & (ТАAAA) 10 & 20.045 \\
\hline DYS632 & $\begin{array}{l}\text { VIC-GGCCGTTGCAAAATAAACTG } \\
\text { gtttcttTCTGGGCAACAGAAGGAGAC }\end{array}$ & AC006371 (nt153697...153732) & $($ CATT $) 9$ & 14.347 \\
\hline $\begin{array}{l}\text { DYS635 } \\
\quad(\text { GATA-C4) }\end{array}$ & Yfiler kit (Applied Biosystems) used & $\begin{array}{l}\text { AC004772 (reverse \& } \\
\text { complement) }\end{array}$ & $\begin{array}{l}{[\text { TCTA }]_{4}(\text { TGTA })_{2}} \\
{[\text { TCTA }]_{2}(\text { TGTA })_{2}} \\
{[\text { TCTA }]_{2}(\text { TGTA })_{2}} \\
{[\text { TCTA }]_{9}=\mathbf{2 3}}\end{array}$ & 13.690 \\
\hline DYS641 & $\begin{array}{l}\text { NED-CTTGAGCCCAGGAAGCATAG } \\
\text { gtttcttCCACACGATGCAATTTTGTC }\end{array}$ & AC018677 (nt3842...3881) & (TAAA) 10 & 14.572 \\
\hline DYS643 & $\begin{array}{l}\text { FAM-AAGCCATGCCTGGTTAAACT } \\
\text { gtttcttTGTAACCAAACACCACCCATT }\end{array}$ & AC007007 (nt25665...25719) & (CTTTT)11 & 15.864 \\
\hline
\end{tabular}


BLAT (http://genome.ucsc.edu/cgi-bin/hgBlat) and are based on the July 2003 assembly of the human genome. Primers were crosschecked against one another using the AutoDimer program [4] to ensure that only compatible loci were combined with one another in testing.

PCR amplification: For each sample, the Y-STR markers were typed in small multiplexes containing 3,4 , or 5 loci. For example, the 20 loci examined in most detail were run in five sets: (a) DYS495, DYS508, DYS520, and DYS533; (b) DYS505, DYS540, DYS522, and DYS532; (c) DYS485, DYS556, DYS594, and DYS449; (d) DYS576, DYS463, DYS570, and DYS446; (e) DYS643, DYS557, DYS534, and DYS444. Amplifications were performed in reaction volumes of $10 \mu \mathrm{L}$ using a master mix containing $1 \mathrm{X}$ GeneAmp PCR Gold buffer (Applied Biosystems, Foster City, CA), $1.5 \mathrm{mmol} / \mathrm{L} \mathrm{MgCl} 2,250 \mu \mathrm{mol} / \mathrm{L}$ deoxynucleotide triphosphates (dNTPs; Promega Corporation, Madison, WI), $0.16 \mathrm{mg} / \mathrm{mL}$ bovine serum albumin (BSA) fraction $\mathrm{V}$ (Sigma, St. Louis, MO), and 1.0 unit of AmpliTaq Gold DNA polymerase (Applied Biosystems). The thermal cycling program was carried out on a GeneAmp 9700 (Applied Biosystems) using the following conditions in 9600-emulation mode (i.e., ramp speeds of $1{ }^{\circ} \mathrm{C} / \mathrm{s}$ ): $95^{\circ} \mathrm{C}$ for $10 \mathrm{~min} ; 30$ cycles of $\left\{95^{\circ} \mathrm{C}\right.$ for $1 \mathrm{~min}, 55^{\circ} \mathrm{C}$ for $1 \mathrm{~min}$, $72{ }^{\circ} \mathrm{C}$ for $\left.1 \mathrm{~min}\right\} ; 60{ }^{\circ} \mathrm{C}$ for $45 \mathrm{~min}$; and $25^{\circ} \mathrm{C}$ until removed from thermal cycler.

Electrophoresis and typing: A $1.0 \mu \mathrm{L}$ aliquot of each Y-STR PCR product was diluted in $14 \mu \mathrm{L} \mathrm{Hi-Di}{ }^{\mathrm{TM}}$ formamide and $0.4 \mu \mathrm{L}$ GS500-LIZ internal size standard (Applied Biosystems) and analyzed on the 16-capillary ABI Prism 3100 Genetic Analyzer (Applied Biosystems) using filter set G5 without prior denaturation of samples. Samples were injected electrokinetically for $10 \mathrm{~s}$ at $3 \mathrm{kV}$. Separations were performed in approximately $60 \mathrm{~min}$ on a $36 \mathrm{~cm}$ array using $\mathrm{POP}^{\mathrm{TM}}$-6 (Applied Biosystems). Automated allele sizing was performed with Genotyper 3.7.

DNA sequencing: At least two different alleles were sequenced for each Y-STR locus with the exception of DYS575 and DYS641 to aid calibration of observed size to repeat number and to assist in nomenclature decisions. Unlabeled primers (see Table 1) were used for generating both forward and reverse sequencing reactions. Thermal cycling was performed using conditions described above but with 35 cycles instead of 30 . Amplicon purity and concentration were assessed with the Agilent BioAnalyzer 2100 (Agilent Technologies, Palo Alto, CA). Following this assessment, unincorporated primers and dNTPs were removed by adding $2 \mu \mathrm{L}$ of ExoSAP-IT (USB Corp., Cleveland, $\mathrm{OH}$ ) to each $5 \mu \mathrm{L}$ PCR reaction. Reactions were mixed briefly and incubated at $37^{\circ} \mathrm{C}$ for $15 \mathrm{~min}$ and then $80^{\circ} \mathrm{C}$ for $15 \mathrm{~min}$ to inactive the enzymes. Cycle sequencing was performed with the ABI PRISM BigDye Terminator (Version 3.1) cycle sequencing kit (Applied Biosystems) using $2 \mu \mathrm{L}$ 5X Sequencing Buffer (Applied Biosystems), $4 \mu \mathrm{L}$ BigDye Terminator kit mix (Applied Biosystems), $3.2 \mu \mathrm{L}$ of $1 \mu \mathrm{mol} / \mathrm{L}$ forward or reverse primer, and Exo-SAP treated
PCR product plus sterile water to bring the total reaction volume to $20 \mu \mathrm{L}$. The target quantity for each PCR product in the sequencing reaction was approximately $7 \mathrm{ng}$ based on the Agilent 2100 determined value. Thermal cycling was conducted in a GeneAmp 9700 thermocycler with the following conditions: an initial $1 \mathrm{~min}$ denaturation at $96{ }^{\circ} \mathrm{C}$; followed by 25 cycles of $15 \mathrm{~s}$ at $94{ }^{\circ} \mathrm{C}$ (denaturation), $5 \mathrm{~s}$ at $50^{\circ} \mathrm{C}$ (annealing), and $2 \mathrm{~min}$ at $60^{\circ} \mathrm{C}$ (extension). The DNA product was purified by filtration though a single tube cartridge or 96-well plate DTR filtration system (Edge BioSystems, Gaithersburg, MD). Purified sequencing products were separated on an ABI 3100 Genetic Analyzer using POP-6 polymer. Sequences were aligned with the GenBank reference allele (accession numbers listed in Table 1) and edited using Sequencher Plus 4.0.5b11 (GeneCodes, Ann Arbor, MI).

Y-STR allele nomenclature: The repeat structure used to define the nomenclature for each Y-STR marker is given in Table 1. The repeat motifs for each of the markers were determined using guidance provided by the DNA Commission of International Society for Forensic Genetics (ISFG) [5].

Analysis of data: Allele frequencies were estimated by direct counting. Gene diversities $(D)$ were calculated according to $D=(n / n-1)\left(1-\Sigma p_{i}^{2}\right)$, where $n$ is the sample size and $p_{i}$ is the allelic frequency [6].

Access to the data: Genotyping results for these Y-STRs are posted at http://www.cstl.nist.gov/biotech/strbase/ NISTpop.htm.

Results and discussion: This data set provides a continuation of information made available on these same samples by Schoske et al. [7], where results from 27 amplified regions of the Y-chromosome were reported. In addition, a concordance study with a beta-test version of Applied Biosystems' Yfiler kit found that there was complete agreement with the Schoske et al. [7] data with the exception of a nomenclature difference in DYS448. The nomenclature by Redd et al. [8] for DYS448 is now recommended and part of the certified values for the NIST Human Y-chromosome DNA Profiling Standard Reference Material SRM 2395 (see http://ts.nist.gov/ts/htdocs/230/ 232/232.htm, then enter 2395). The DYS635 information collected from the Yfiler testing of our U.S. population samples is presented in Table 2 along with the other new Y-STR loci examined as part of this study.

Most of the loci examined in the present study were selected from the most polymorphic simple repeats reported by Kayser et al. [9] in their comprehensive survey of the Y-chromosome. Since the Kayser et al. [9] survey only examined eight different samples albeit from diverse backgrounds, the information collected as part of this study represents a first attempt to aid assessment of these loci in populations of interest through collection of allele frequency information and thus help establish which loci are most polymorphic and desirable to pursue in future population studies and human identity applications. It should be noted that some allele frequency information has been 
Table 2

The distribution of allele frequencies observed in U.S. Caucasian (Cau), African American (AA), and Hispanic (His) sample sets listed by Y-STR locus (see Table 1)

\begin{tabular}{|c|c|c|c|c|c|}
\hline Locus & Allele & Combined frequency $(N=654)$ & Cau frequency $(N=258)$ & AA frequency $(N=257)$ & His frequency $(N=139)$ \\
\hline \multirow[t]{7}{*}{ DYS444 } & 10 & 0.0107 & 0.0039 & 0.0117 & 0.0216 \\
\hline & 11 & 0.1101 & 0.0659 & 0.1206 & 0.1727 \\
\hline & 12 & 0.5872 & 0.5930 & 0.6304 & 0.4964 \\
\hline & 13 & 0.2202 & 0.2597 & 0.1712 & 0.2374 \\
\hline & 14 & 0.0596 & 0.0698 & 0.0545 & 0.0504 \\
\hline & 15 & 0.0107 & 0.0078 & 0.0117 & 0.0144 \\
\hline & 16 & 0.0015 & 0.0000 & 0.0000 & 0.0072 \\
\hline Locus & Allele & Combined frequency $(N=660)$ & Cau frequency $(N=262)$ & AA frequency $(N=258)$ & His frequency $(N=140)$ \\
\hline \multirow[t]{14}{*}{ DYS446 } & 8 & 0.0015 & 0.0000 & 0.0000 & 0.0071 \\
\hline & 10 & 0.0061 & 0.0115 & 0.0000 & 0.0071 \\
\hline & 11 & 0.0318 & 0.0420 & 0.0310 & 0.0143 \\
\hline & 12 & 0.1576 & 0.1450 & 0.1357 & 0.2214 \\
\hline & 13 & 0.5121 & 0.5763 & 0.4767 & 0.4571 \\
\hline & 14 & 0.1682 & 0.1450 & 0.1938 & 0.1643 \\
\hline & 15 & 0.0545 & 0.0382 & 0.0736 & 0.0500 \\
\hline & 15.1 & 0.0015 & 0.0000 & 0.0000 & 0.0071 \\
\hline & 16 & 0.0288 & 0.0076 & 0.0465 & 0.0357 \\
\hline & 17 & 0.0152 & 0.0076 & 0.0155 & 0.0286 \\
\hline & 18 & 0.0076 & 0.0076 & 0.0116 & 0.0000 \\
\hline & 19 & 0.0091 & 0.0115 & 0.0078 & 0.0071 \\
\hline & 20 & 0.0030 & 0.0038 & 0.0039 & 0.0000 \\
\hline & 21 & 0.0030 & 0.0038 & 0.0039 & 0.0000 \\
\hline Locus & Allele & Combined frequency $(N=651)$ & Cau frequency $(N=261)$ & AA frequency $(N=253)$ & His frequency $(N=137)$ \\
\hline \multirow{14}{*}{ DYS449 } & 24 & 0.0015 & 0.0038 & 0.0000 & 0.0000 \\
\hline & 25 & 0.0061 & 0.0038 & 0.0000 & 0.0219 \\
\hline & 26 & 0.0077 & 0.0115 & 0.0040 & 0.0073 \\
\hline & 27 & 0.0445 & 0.0421 & 0.0553 & 0.0292 \\
\hline & 28 & 0.1459 & 0.1724 & 0.1186 & 0.1460 \\
\hline & 29 & 0.2611 & 0.2989 & 0.1818 & 0.3358 \\
\hline & 30 & 0.2043 & 0.2529 & 0.1779 & 0.1606 \\
\hline & 31 & 0.1536 & 0.1149 & 0.2016 & 0.1387 \\
\hline & 32 & 0.1045 & 0.0651 & 0.1542 & 0.0876 \\
\hline & 33 & 0.0369 & 0.0230 & 0.0435 & 0.0511 \\
\hline & 34 & 0.0123 & 0.0038 & 0.0198 & 0.0146 \\
\hline & 35 & 0.0108 & 0.0038 & 0.0237 & 0.0000 \\
\hline & 36 & 0.0077 & 0.0038 & 0.0119 & 0.0073 \\
\hline & 37 & 0.0031 & 0.0000 & 0.0079 & 0.0000 \\
\hline Locus & Allele & Combined frequency $(N=661)$ & Cau frequency $(N=262)$ & AA frequency $(N=258)$ & His frequency $(N=140)$ \\
\hline \multirow[t]{11}{*}{ DYS463 } & 17 & 0.0015 & 0.0038 & 0.0000 & 0.0000 \\
\hline & 18 & 0.0408 & 0.0304 & 0.0194 & 0.1000 \\
\hline & 19 & 0.0106 & 0.0038 & 0.0233 & 0.0000 \\
\hline & 20 & 0.2284 & 0.0076 & 0.5388 & 0.0714 \\
\hline & 21 & 0.1014 & 0.1331 & 0.0969 & 0.0500 \\
\hline & 22 & 0.1120 & 0.1483 & 0.0465 & 0.1643 \\
\hline & 23 & 0.0530 & 0.1027 & 0.0116 & 0.0357 \\
\hline & 24 & 0.3873 & 0.4905 & 0.2287 & 0.4857 \\
\hline & 25 & 0.0560 & 0.0722 & 0.0310 & 0.0714 \\
\hline & 26 & 0.0076 & 0.0076 & 0.0000 & 0.0214 \\
\hline & 28 & 0.0015 & 0.0000 & 0.0039 & 0.0000 \\
\hline Locus & Allele & Combined frequency $(N=655)$ & Cau frequency $(N=262)$ & AA frequency $(N=254)$ & His frequency $(N=139)$ \\
\hline \multirow[t]{2}{*}{ DYS485 } & 10 & 0.0061 & 0.0000 & 0.0157 & 0.0000 \\
\hline & 12 & 0.0702 & 0.1298 & 0.0276 & 0.0360 \\
\hline
\end{tabular}


Table 2 (Continued)

\begin{tabular}{|c|c|c|c|c|c|}
\hline Locus & Allele & Combined frequency $(N=655)$ & Cau frequency $(N=262)$ & AA frequency $(N=254)$ & His frequency $(N=139)$ \\
\hline & 13 & 0.0244 & 0.0344 & 0.0118 & 0.0288 \\
\hline & 14 & 0.2824 & 0.0992 & 0.5197 & 0.1942 \\
\hline & 15 & 0.5084 & 0.6336 & 0.2992 & 0.6547 \\
\hline & 16 & 0.0809 & 0.0649 & 0.1102 & 0.0576 \\
\hline & 17 & 0.0229 & 0.0267 & 0.0157 & 0.0288 \\
\hline & 18 & 0.0046 & 0.0115 & 0.0000 & 0.0000 \\
\hline Locus & Allele & Combined frequency $(N=92)$ & Cau frequency $(N=29)$ & AA frequency $(N=31)$ & His frequency $(N=32)$ \\
\hline \multirow[t]{18}{*}{ DYS490 } & 8,13 & 0.0435 & 0.0000 & 0.1290 & 0.0000 \\
\hline & 9,12 & 0.0978 & 0.0690 & 0.1290 & 0.0938 \\
\hline & 9,13 & 0.0326 & 0.0000 & 0.0645 & 0.0313 \\
\hline & 9,14 & 0.0109 & 0.0000 & 0.0000 & 0.0313 \\
\hline & 9,15 & 0.0109 & 0.0000 & 0.0323 & 0.0000 \\
\hline & 9,16 & 0.0109 & 0.0000 & 0.0323 & 0.0000 \\
\hline & 10,13 & 0.0109 & 0.0000 & 0.0323 & 0.0000 \\
\hline & 12,13 & 0.0109 & 0.0000 & 0.0000 & 0.0313 \\
\hline & 12,14 & 0.3804 & 0.5172 & 0.1290 & 0.5000 \\
\hline & 12,15 & 0.0217 & 0.0345 & 0.0000 & 0.0313 \\
\hline & 12,16 & 0.1304 & 0.1379 & 0.0323 & 0.2188 \\
\hline & 12,17 & 0.0652 & 0.1724 & 0.0323 & 0.0000 \\
\hline & 12,18 & 0.0217 & 0.0690 & 0.0000 & 0.0000 \\
\hline & 13,13 & 0.0217 & 0.0000 & 0.0645 & 0.0000 \\
\hline & 13,14 & 0.0870 & 0.0000 & 0.2258 & 0.0313 \\
\hline & 13,15 & 0.0109 & 0.0000 & 0.0323 & 0.0000 \\
\hline & 13,16 & 0.0217 & 0.0000 & 0.0323 & 0.0313 \\
\hline & 14,15 & 0.0109 & 0.0000 & 0.0323 & 0.0000 \\
\hline Locus & Allele & Combined frequency $(N=659)$ & Cau frequency $(N=262)$ & AA frequency $(N=257)$ & His frequency $(N=140)$ \\
\hline \multirow[t]{7}{*}{ DYS495 } & 12 & 0.0076 & 0.0000 & 0.0039 & 0.0286 \\
\hline & 13 & 0.0076 & 0.0000 & 0.0039 & 0.0286 \\
\hline & 14 & 0.0455 & 0.0725 & 0.0156 & 0.0500 \\
\hline & 15 & 0.3748 & 0.1870 & 0.6070 & 0.3000 \\
\hline & 16 & 0.4446 & 0.5725 & 0.2802 & 0.5071 \\
\hline & 17 & 0.1153 & 0.1641 & 0.0856 & 0.0786 \\
\hline & 18 & 0.0046 & 0.0038 & 0.0039 & 0.0071 \\
\hline Locus & Allele & Combined frequency $(N=94)$ & Cau frequency $(N=30)$ & AA frequency $(N=32)$ & His frequency $(N=32)$ \\
\hline \multirow[t]{9}{*}{ DYS504 } & 11 & 0.0213 & 0.0000 & 0.0313 & 0.0313 \\
\hline & 12 & 0.0213 & 0.0000 & 0.0625 & 0.0000 \\
\hline & 13 & 0.2128 & 0.0000 & 0.4688 & 0.1563 \\
\hline & 14 & 0.1489 & 0.1667 & 0.1250 & 0.1563 \\
\hline & 15 & 0.0638 & 0.0667 & 0.0313 & 0.0938 \\
\hline & 16 & 0.1702 & 0.2333 & 0.0625 & 0.2188 \\
\hline & 17 & 0.3085 & 0.4667 & 0.2188 & 0.2500 \\
\hline & 18 & 0.0426 & 0.0667 & 0.0000 & 0.0625 \\
\hline & 19 & 0.0106 & 0.0000 & 0.0000 & 0.0313 \\
\hline Locus & Allele & Combined frequency $(N=654)$ & Cau frequency $(N=262)$ & AA frequency $(N=253)$ & His frequency $(N=139)$ \\
\hline \multirow[t]{7}{*}{ DYS505 } & 9 & 0.0076 & 0.0115 & 0.0000 & 0.0144 \\
\hline & 10 & 0.0031 & 0.0038 & 0.0040 & 0.0000 \\
\hline & 11 & 0.2232 & 0.2366 & 0.1818 & 0.2734 \\
\hline & 12 & 0.4541 & 0.5000 & 0.3874 & 0.4892 \\
\hline & 13 & 0.2783 & 0.2290 & 0.3676 & 0.2086 \\
\hline & 14 & 0.0275 & 0.0191 & 0.0435 & 0.0144 \\
\hline & 15 & 0.0061 & 0.0000 & 0.0158 & 0.0000 \\
\hline Locus & Allele & Combined frequency $(N=658)$ & Cau frequency $(N=261)$ & AA frequency $(N=257)$ & His frequency $(N=140)$ \\
\hline \multirow[t]{2}{*}{ DYS508 } & 8 & 0.0015 & 0.0038 & 0.0000 & 0.0000 \\
\hline & 9 & 0.0015 & 0.0038 & 0.0000 & 0.0000 \\
\hline
\end{tabular}


Table 2 (Continued)

\begin{tabular}{|c|c|c|c|c|c|}
\hline Locus & Allele & Combined frequency $(N=658)$ & Cau frequency $(N=261)$ & AA frequency $(N=257)$ & His frequency $(N=140)$ \\
\hline & 10 & 0.1292 & 0.2146 & 0.0506 & 0.1143 \\
\hline & 10.1 & 0.0015 & 0.0038 & 0.0000 & 0.0000 \\
\hline & 11 & 0.4833 & 0.6207 & 0.3074 & 0.5500 \\
\hline & 12 & 0.2325 & 0.1264 & 0.3385 & 0.2357 \\
\hline & 13 & 0.0638 & 0.0192 & 0.1206 & 0.0429 \\
\hline & 14 & 0.0608 & 0.0077 & 0.1206 & 0.0500 \\
\hline & 15 & 0.0258 & 0.0000 & 0.0623 & 0.0071 \\
\hline Locus & Allele & Combined frequency $(N=659)$ & Cau frequency $(N=262)$ & AA frequency $(N=257)$ & His frequency $(N=140)$ \\
\hline \multirow[t]{9}{*}{ DYS520 } & 18 & 0.0061 & 0.0115 & 0.0039 & 0.0000 \\
\hline & 19 & 0.0395 & 0.0382 & 0.0156 & 0.0857 \\
\hline & 20 & 0.0425 & 0.0611 & 0.0156 & 0.0571 \\
\hline & 21 & 0.4856 & 0.6450 & 0.2918 & 0.5429 \\
\hline & 22 & 0.1608 & 0.1718 & 0.1479 & 0.1643 \\
\hline & 23 & 0.2231 & 0.0687 & 0.4280 & 0.1357 \\
\hline & 24 & 0.0319 & 0.0038 & 0.0700 & 0.0143 \\
\hline & 25 & 0.0091 & 0.0000 & 0.0233 & 0.0000 \\
\hline & 26 & 0.0015 & 0.0000 & 0.0039 & 0.0000 \\
\hline Locus & Allele & Combined frequency $(N=655)$ & Cau frequency $(N=259)$ & AA frequency $(N=258)$ & His frequency $(N=138)$ \\
\hline \multirow[t]{8}{*}{ DYS522 } & 8 & 0.0015 & 0.0039 & 0.0000 & 0.0000 \\
\hline & 9 & 0.0031 & 0.0039 & 0.0000 & 0.0072 \\
\hline & 10 & 0.3099 & 0.3707 & 0.2093 & 0.3841 \\
\hline & 11 & 0.4519 & 0.4208 & 0.5233 & 0.3768 \\
\hline & 12 & 0.2015 & 0.1815 & 0.2248 & 0.1957 \\
\hline & 13 & 0.0290 & 0.0154 & 0.0426 & 0.0290 \\
\hline & 14 & 0.0015 & 0.0000 & 0.0000 & 0.0072 \\
\hline & 17 & 0.0015 & 0.0039 & 0.0000 & 0.0000 \\
\hline Locus & Allele & Combined frequency $(N=93)$ & Cau frequency $(N=29)$ & AA frequency $(N=32)$ & His frequency $(N=32)$ \\
\hline \multirow[t]{7}{*}{ DYS525 } & 7 & 0.0108 & 0.0000 & 0.0313 & 0.0000 \\
\hline & 8 & 0.0108 & 0.0000 & 0.0313 & 0.0000 \\
\hline & 9 & 0.0538 & 0.0000 & 0.0000 & 0.1563 \\
\hline & 10 & 0.6774 & 0.7586 & 0.7813 & 0.5000 \\
\hline & 11 & 0.1505 & 0.2069 & 0.0625 & 0.1875 \\
\hline & 12 & 0.0645 & 0.0000 & 0.0938 & 0.0938 \\
\hline & 13 & 0.0323 & 0.0345 & 0.0000 & 0.0625 \\
\hline Locus & Allele & Combined frequency $(N=655)$ & Cau frequency $(N=262)$ & AA frequency $(N=254)$ & His frequency $(N=139)$ \\
\hline \multirow[t]{9}{*}{ DYS532 } & 9 & 0.0153 & 0.0267 & 0.0039 & 0.0144 \\
\hline & 10 & 0.0443 & 0.0305 & 0.0433 & 0.0719 \\
\hline & 11 & 0.1252 & 0.1641 & 0.0945 & 0.1079 \\
\hline & 12 & 0.2290 & 0.1489 & 0.3150 & 0.2230 \\
\hline & 13 & 0.3588 & 0.3893 & 0.3425 & 0.3309 \\
\hline & 14 & 0.1450 & 0.1603 & 0.1181 & 0.1655 \\
\hline & 15 & 0.0626 & 0.0649 & 0.0591 & 0.0647 \\
\hline & 16 & 0.0168 & 0.0153 & 0.0197 & 0.0144 \\
\hline & 17 & 0.0031 & 0.0000 & 0.0039 & 0.0072 \\
\hline Locus & Allele & Combined frequency $(N=660)$ & Cau frequency $(N=262)$ & AA frequency $(N=258)$ & His frequency $(N=140)$ \\
\hline \multirow[t]{7}{*}{ DYS533 } & 9 & 0.0258 & 0.0305 & 0.0078 & 0.0500 \\
\hline & 10 & 0.0333 & 0.0115 & 0.0543 & 0.0357 \\
\hline & 11 & 0.3424 & 0.2023 & 0.5155 & 0.2857 \\
\hline & 12 & 0.4848 & 0.5954 & 0.3295 & 0.5643 \\
\hline & 13 & 0.0864 & 0.1260 & 0.0698 & 0.0429 \\
\hline & 14 & 0.0258 & 0.0305 & 0.0233 & 0.0214 \\
\hline & 12,13 & 0.0015 & 0.0038 & 0.0000 & 0.0000 \\
\hline
\end{tabular}


Table 2 (Continued)

\begin{tabular}{|c|c|c|c|c|c|}
\hline Locus & Allele & Combined frequency $(N=657)$ & Cau frequency $(N=261)$ & AA frequency $(N=256)$ & His frequency $(N=140)$ \\
\hline \multirow[t]{13}{*}{ DYS534 } & 10 & 0.0015 & 0.0000 & 0.0039 & 0.0000 \\
\hline & 11 & 0.0030 & 0.0038 & 0.0039 & 0.0000 \\
\hline & 12 & 0.0091 & 0.0038 & 0.0195 & 0.0000 \\
\hline & 13 & 0.0487 & 0.0421 & 0.0430 & 0.0714 \\
\hline & 14 & 0.1339 & 0.1533 & 0.1445 & 0.0786 \\
\hline & 15 & 0.3805 & 0.3525 & 0.4141 & 0.3714 \\
\hline & 15.1 & 0.0030 & 0.0077 & 0.0000 & 0.0000 \\
\hline & 16 & 0.2542 & 0.2644 & 0.2383 & 0.2643 \\
\hline & 17 & 0.1187 & 0.1111 & 0.1016 & 0.1643 \\
\hline & 18 & 0.0381 & 0.0498 & 0.0273 & 0.0357 \\
\hline & 19 & 0.0061 & 0.0038 & 0.0039 & 0.0143 \\
\hline & 20 & 0.0015 & 0.0038 & 0.0000 & 0.0000 \\
\hline & 15,17 & 0.0015 & 0.0038 & 0.0000 & 0.0000 \\
\hline Locus & Allele & Combined frequency $(N=659)$ & Cau frequency $(N=263)$ & AA frequency $(N=257)$ & His frequency $(N=139)$ \\
\hline \multirow{5}{*}{ DYS540 } & 10 & 0.0137 & 0.0076 & 0.0195 & 0.0144 \\
\hline & 11 & 0.2200 & 0.1787 & 0.2062 & 0.3237 \\
\hline & 12 & 0.7132 & 0.7567 & 0.7160 & 0.6259 \\
\hline & 13 & 0.0501 & 0.0532 & 0.0545 & 0.0360 \\
\hline & 14 & 0.0030 & 0.0038 & 0.0039 & 0.0000 \\
\hline Locus & Allele & Combined frequency $(N=655)$ & Cau frequency $(N=262)$ & AA frequency $(N=254)$ & His frequency $(N=139)$ \\
\hline \multirow{6}{*}{ DYS556 } & 9 & 0.0031 & 0.0038 & 0.0039 & 0.0000 \\
\hline & 10 & 0.0076 & 0.0153 & 0.0039 & 0.0000 \\
\hline & 10.1 & 0.0015 & 0.0038 & 0.0000 & 0.0000 \\
\hline & 11 & 0.5863 & 0.7214 & 0.3937 & 0.6835 \\
\hline & 12 & 0.3603 & 0.2328 & 0.5276 & 0.2950 \\
\hline & 13 & 0.0412 & 0.0229 & 0.0709 & 0.0216 \\
\hline Locus & Allele & Combined frequency $(N=658)$ & Cau frequency $(N=262)$ & AA frequency $(N=257)$ & His frequency $(N=139)$ \\
\hline \multirow[t]{12}{*}{ DYS557 } & 11 & 0.0015 & 0.0038 & 0.0000 & 0.0000 \\
\hline & 12 & 0.0015 & 0.0000 & 0.0000 & 0.0071 \\
\hline & 13 & 0.0030 & 0.0000 & 0.0038 & 0.0071 \\
\hline & 14 & 0.0441 & 0.0830 & 0.0154 & 0.0214 \\
\hline & 15 & 0.1520 & 0.2302 & 0.0654 & 0.1571 \\
\hline & 16 & 0.3754 & 0.4566 & 0.2462 & 0.4429 \\
\hline & 17 & 0.1717 & 0.1396 & 0.1923 & 0.1857 \\
\hline & 18 & 0.1672 & 0.0604 & 0.2923 & 0.1286 \\
\hline & 19 & 0.0593 & 0.0113 & 0.1231 & 0.0286 \\
\hline & 20 & 0.0167 & 0.0038 & 0.0308 & 0.0143 \\
\hline & 21 & 0.0061 & 0.0000 & 0.0154 & 0.0000 \\
\hline & 22 & 0.0015 & 0.0000 & 0.0038 & 0.0000 \\
\hline Locus & Allele & Combined frequency $(N=661)$ & Cau frequency $(N=262)$ & AA frequency $(N=259)$ & His frequency $(N=140)$ \\
\hline \multirow[t]{12}{*}{ DYS570 } & 12 & 0.0015 & 0.0000 & 0.0039 & 0.0000 \\
\hline & 13 & 0.0015 & 0.0000 & 0.0039 & 0.0000 \\
\hline & 14 & 0.0076 & 0.0000 & 0.0193 & 0.0000 \\
\hline & 15 & 0.0197 & 0.0153 & 0.0309 & 0.0071 \\
\hline & 16 & 0.0893 & 0.0878 & 0.0618 & 0.1429 \\
\hline & 17 & 0.3404 & 0.4084 & 0.2934 & 0.3000 \\
\hline & 18 & 0.2390 & 0.2405 & 0.2548 & 0.2071 \\
\hline & 19 & 0.1679 & 0.1221 & 0.1969 & 0.2000 \\
\hline & 20 & 0.0832 & 0.0878 & 0.1004 & 0.0429 \\
\hline & 21 & 0.0227 & 0.0229 & 0.0193 & 0.0286 \\
\hline & 22 & 0.0212 & 0.0115 & 0.0116 & 0.0571 \\
\hline & 23 & 0.0061 & 0.0038 & 0.0039 & 0.0143 \\
\hline Locus & Allele & Combined frequency $(N=94)$ & Cau frequency $(N=30)$ & AA frequency $(N=32)$ & His frequency $(N=32)$ \\
\hline \multirow[t]{2}{*}{ DYS575 } & 9 & 0.0106 & 0.0000 & 0.0313 & 0.0000 \\
\hline & 10 & 0.9894 & 1.0000 & 0.9688 & 1.0000 \\
\hline
\end{tabular}


Table 2 (Continued)

\begin{tabular}{|c|c|c|c|c|c|}
\hline Locus & Allele & Combined frequency $(N=659)$ & Cau frequency $(N=261)$ & AA frequency $(N=258)$ & His frequency $(N=140)$ \\
\hline \multirow[t]{9}{*}{ DYS576 } & 13 & 0.0030 & 0.0038 & 0.0039 & 0.0000 \\
\hline & 14 & 0.0273 & 0.0115 & 0.0426 & 0.0286 \\
\hline & 15 & 0.1047 & 0.0421 & 0.1977 & 0.0500 \\
\hline & 16 & 0.1745 & 0.1762 & 0.2016 & 0.1214 \\
\hline & 17 & 0.2640 & 0.2835 & 0.2209 & 0.3071 \\
\hline & 18 & 0.2822 & 0.3257 & 0.2403 & 0.2786 \\
\hline & 19 & 0.1077 & 0.1188 & 0.0698 & 0.1571 \\
\hline & 20 & 0.0288 & 0.0307 & 0.0194 & 0.0429 \\
\hline & 21 & 0.0076 & 0.0077 & 0.0039 & 0.0143 \\
\hline Locus & Allele & Combined frequency $(N=657)$ & Cau frequency $(N=262)$ & AA frequency $(N=256)$ & His frequency $(N=139)$ \\
\hline \multirow[t]{6}{*}{ DYS594 } & 9 & 0.0137 & 0.0153 & 0.0156 & 0.0072 \\
\hline & 10 & 0.5693 & 0.8015 & 0.3047 & 0.6187 \\
\hline & 11 & 0.3714 & 0.1756 & 0.5977 & 0.3237 \\
\hline & 12 & 0.0335 & 0.0076 & 0.0586 & 0.0360 \\
\hline & 13 & 0.0091 & 0.0000 & 0.0195 & 0.0072 \\
\hline & 14 & 0.0030 & 0.0000 & 0.0039 & 0.0072 \\
\hline Locus & Allele & Combined frequency $(N=94)$ & Cau frequency $(N=30)$ & AA frequency $(N=32)$ & His frequency $(N=32)$ \\
\hline \multirow[t]{2}{*}{ DYS632 } & 9 & 0.5426 & 0.2333 & 0.8438 & 0.5313 \\
\hline & 10 & 0.4574 & 0.7667 & 0.1563 & 0.4688 \\
\hline Locus & Allele & Combined frequency $(N=661)$ & Cau frequency $(N=263)$ & AA frequency $(N=259)$ & His frequency $(N=139)$ \\
\hline DYS635 & 17 & 0.0121 & 0.0000 & 0.0270 & 0.0072 \\
\hline \multirow[t]{10}{*}{ GATA-C4 } & 19 & 0.0061 & 0.0000 & 0.0154 & 0.0000 \\
\hline & 20 & 0.0575 & 0.0494 & 0.0463 & 0.0935 \\
\hline & 21 & 0.2753 & 0.1559 & 0.4324 & 0.2086 \\
\hline & 21.3 & 0.0015 & 0.0000 & 0.0000 & 0.0072 \\
\hline & 22 & 0.1180 & 0.0989 & 0.1274 & 0.1367 \\
\hline & 23 & 0.3964 & 0.5095 & 0.2471 & 0.4604 \\
\hline & 24 & 0.1089 & 0.1407 & 0.0965 & 0.0719 \\
\hline & 25 & 0.0197 & 0.0380 & 0.0077 & 0.0072 \\
\hline & 26 & 0.0030 & 0.0076 & 0.0000 & 0.0000 \\
\hline & 27 & 0.0015 & 0.0000 & 0.0000 & 0.0072 \\
\hline Locus & Allele & Combined frequency $(N=94)$ & Cau frequency $(N=30)$ & AA frequency $(N=32)$ & His frequency $(N=32)$ \\
\hline \multirow[t]{3}{*}{ DYS641 } & 9 & 0.0106 & 0.0000 & 0.0313 & 0.0000 \\
\hline & 10 & 0.9362 & 0.9333 & 0.8750 & 1.0000 \\
\hline & 11 & 0.0532 & 0.0667 & 0.0938 & 0.0000 \\
\hline Locus & Allele & Combined frequency $(N=657)$ & Cau frequency $(N=263)$ & AA frequency $(N=257)$ & His frequency $(N=137)$ \\
\hline \multirow[t]{10}{*}{ DYS643 } & 7 & 0.0046 & 0.0000 & 0.0078 & 0.0073 \\
\hline & 8 & 0.0046 & 0.0076 & 0.0039 & 0.0000 \\
\hline & 9 & 0.0594 & 0.0798 & 0.0233 & 0.0876 \\
\hline & 10 & 0.4125 & 0.5741 & 0.2062 & 0.4891 \\
\hline & 11 & 0.1065 & 0.1217 & 0.0817 & 0.1241 \\
\hline & 11.1 & 0.0015 & 0.0038 & 0.0000 & 0.0000 \\
\hline & 12 & 0.1613 & 0.1483 & 0.1518 & 0.2044 \\
\hline & 13 & 0.1766 & 0.0608 & 0.3580 & 0.0584 \\
\hline & 14 & 0.0563 & 0.0038 & 0.1245 & 0.0292 \\
\hline & 15 & 0.0167 & 0.0000 & 0.0428 & 0.0000 \\
\hline
\end{tabular}

The most frequent allele is bolded for each group.

previously described with DYS444 [10], DYS446 [8], DYS449 [8], and DYS463 [8]. In addition, Dai et al. [11] recently published DYS520 allele frequencies from a Chinese population. The allele frequencies for DYS446, DYS449, and DYS463 observed for the Caucasian samples in this study were quite similar to those reported for 148 Caucasians in Redd et al. [8] (data not shown).

Table 2 contains the observed allele frequencies for U.S. Caucasian, African American, and Hispanic samples. Note that 21 of the loci were tested across more than 650 total 


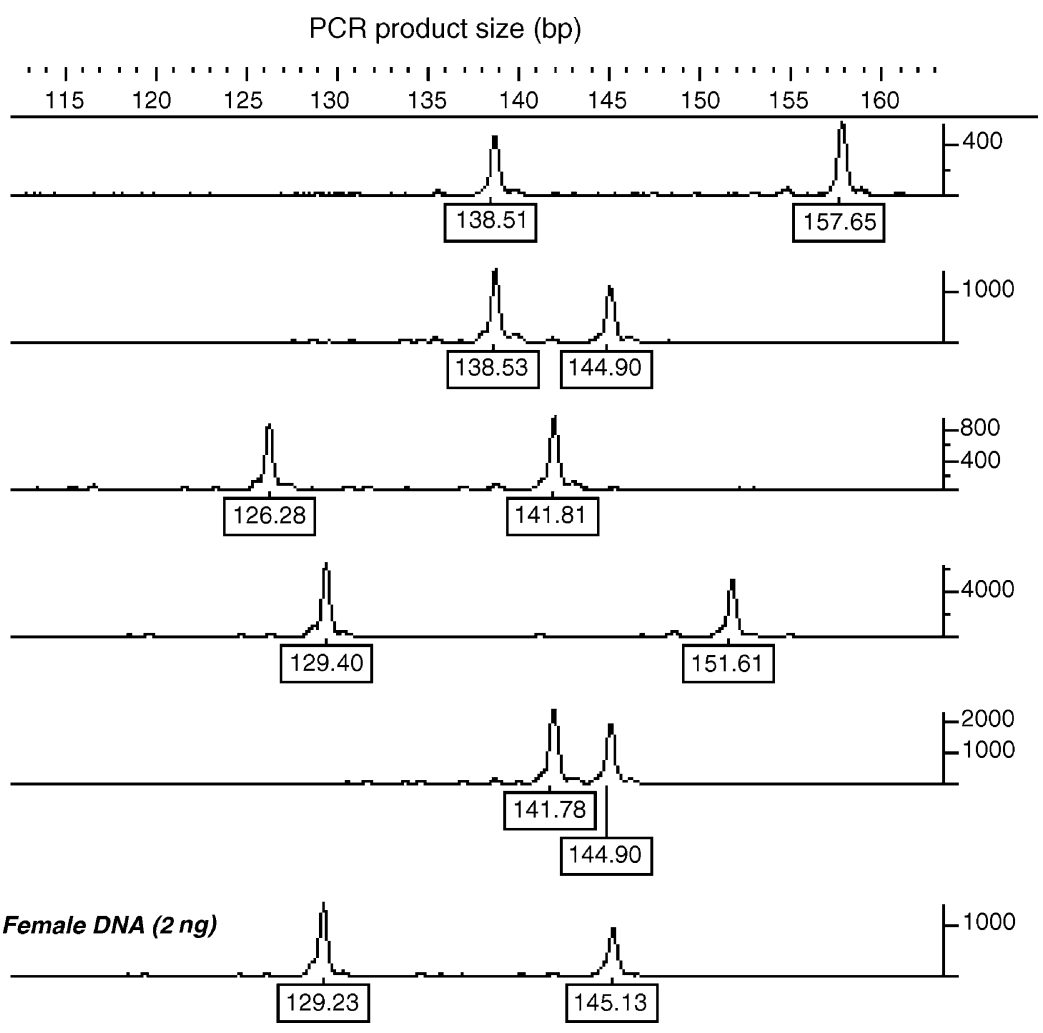

Fig. 1. Various allele patterns observed with the duplicated trinucleotide repeat locus DYS490 in multiple males and a single female sample. All DNA templates were approximately 1-2 ng in amount. Peak labels are PCR product sizes in base pairs (bp) relative to the GS500 LIZ size standard on an ABI 3100.

samples while DYS490, DYS504, DYS525, DYS575, DYS632, and DYS641 were only examined in 92-94 samples due to low variation, poor performance with the current primers, or potential X-chromosome homology. For example, DYS490 produced double peak patterns in most male samples examined as well as a female control (Fig. 1). Due to its female cross-reactivity, forensic laboratories desiring male-specific amplification will not likely adopt DYS490, even though this X-chromosome homology may be reduced or eliminated through careful primer design in the future. None of the other 26 loci examined in this study produced PCR products with the $2 \mathrm{ng}$ female DNA template tested.

The rest of the loci examined in this study were all singlecopy markers and thus exhibited only a single peak at each locus. However, two PCR products were observed in a single Caucasian sample at DYS533 and DYS534. Table 2 lists these allele pairs in a separate category rather than incorporating them into the overall allele count for the two individual alleles. Since the repeat structures for these two loci are only $190 \mathrm{bp}$ apart it is likely that this entire region near 16.831 megabases on the Y-chromosome has been duplicated within this sample.

Nomenclature of allele designation is important to enable effective communication of information across the DNA typing community. Previous nomenclatures, which were published prior to this study, were followed in Table 1 for DYS444 [10], DYS446 [8], DYS449 [8], DYS463 [8], and DYS635 [12]. The nomenclature for the simple repeat loci, such as DYS485, DYS533, or DYS643, is straightforward and follows ISFG recommendations with the earliest possible repeat motif along the GenBank top strand being designated [5]. An examination of the supplementary material attached to the Kayser et al. [9] publication describing these loci indicates that we are in agreement for most of the loci.

There are, however, four loci with possible alternative nomenclatures to the ones presented in Table 1. These include DYS520, DYS532, DYS534, and DYS557. DYS520 contains a compound repeat that was designed $(\text { GATA })_{m}(\text { CATA })_{n}$ by Dai et al. [11]. We have chosen to call the repeat structure (ATAG) $)_{m}(\text { ATAC })_{n}$ in order to reflect the earliest possible motif and thus follow the ISFG guidelines. In this case, there should not be a difference in the total number of repeats using either approach. With DYS532 there are five adjacent repeats possessing similar structure to the core CTTT repeat. If the full motif pattern of $(\text { CTTT })_{3}(\text { CCTT)(CTTT) })_{14}$ (CTTC) was used, then the GenBank reference allele would be designated 19 repeats rather than the 14 repeats based solely on the core CTTT repeat listed in Table 1. DYS534 contains a compound repeat 
structure around the core CTTT repeat: $(\text { CTTT })_{3}$ (CCTT)(CCTC)(CTTT) ${ }_{15}($ CTGT)(CTTT). We have chosen to only designate the core 15 CTTT repeats rather than 22 $(3+1+1+15+1+1)$ compound repeats. Likewise DYS557 possesses extra TTTC repeats in close proximity to the core TTTC repeat that might be included in an alternative approach to designating the repeat structure. The bottom strand of GenBank accession AC004772 is used to denote DYS635 in order to maintain consistency with previous work, referred to then as Y-GATA-C4 [12].

The variant alleles observed in this study have been confirmed through mixing experiments with adjacent alleles for each locus. The following variant alleles have been sequenced: DYS556 allele 10.1 ([AATA $]_{2}$ AATTA $\left.[\text { AATA }]_{7}\right)$, DYS635 allele 21.3 ([TCTA $]_{4}(\text { TGTA })_{2}[$ TCTA $]_{2}(\mathrm{TGTA})_{2}[\mathrm{TCTA}]_{2}(\mathrm{TGTA})_{2}[\mathrm{TCTA}]_{5}$ TC-A $\left.[\mathrm{TCTA}]_{2}\right)$, and DYS643 allele 11.1 ([CTTTT $\left.]_{8} \underline{\mathrm{C}}[\mathrm{CTTTT}]_{3}\right)$.

The data presented in this work are preliminary. However, it appears that several simple repeat loci including DYS570 and DYS576 are highly polymorphic and thus may aid resolution of common Y-STR haplotypes, which cannot be separated with the widely used minimal haplotype loci. A

Table 3

Comparison of gene diversity values for the 27 Y-STR loci across the U.S. samples examined in this study

\begin{tabular}{|c|c|c|c|c|c|c|}
\hline Locus & $N$ & Diversity & Rank & Caucasian & $\begin{array}{l}\text { African } \\
\text { American }\end{array}$ & Hispanic \\
\hline DYS449 & 651 & 0.8318 & 1 & 0.8001 & 0.8541 & 0.8149 \\
\hline DYS490 & 92 & 0.8201 & 2 & 0.6970 & 0.9118 & 0.7097 \\
\hline DYS504 & 94 & 0.8101 & 3 & 0.7149 & 0.7298 & 0.8528 \\
\hline DYS576 & 661 & 0.7972 & 4 & 0.7684 & 0.8098 & 0.7889 \\
\hline DYS570 & 661 & 0.7836 & 5 & 0.7470 & 0.7975 & 0.8063 \\
\hline DYS532 & 656 & 0.7769 & 6 & 0.7705 & 0.7578 & 0.7976 \\
\hline DYS557 & 658 & 0.7739 & 7 & 0.7110 & 0.7992 & 0.7318 \\
\hline DYS463 & 661 & 0.7684 & 8 & 0.7056 & 0.6464 & 0.7178 \\
\hline DYS534 & 657 & 0.7557 & 9 & 0.7686 & 0.7404 & 0.7579 \\
\hline DYS643 & 657 & 0.7554 & 10 & 0.6258 & 0.7847 & 0.6968 \\
\hline DYS635 & 661 & 0.7385 & 11 & 0.6852 & 0.7260 & 0.7168 \\
\hline DYS508 & 658 & 0.6883 & 12 & 0.5544 & 0.7583 & 0.6290 \\
\hline DYS520 & 659 & 0.6851 & 13 & 0.5465 & 0.7066 & 0.6538 \\
\hline DYS446 & 660 & 0.6804 & 14 & 0.6245 & 0.7105 & 0.7151 \\
\hline DYS505 & 654 & 0.6666 & 15 & 0.6435 & 0.6823 & 0.6466 \\
\hline DYS522 & 656 & 0.6593 & 16 & 0.6548 & 0.6325 & 0.6762 \\
\hline DYS485 & 655 & 0.6501 & 17 & 0.5678 & 0.6293 & 0.5312 \\
\hline DYS495 & 659 & 0.6473 & 18 & 0.6074 & 0.5476 & 0.6471 \\
\hline DYS533 & 660 & 0.6387 & 19 & 0.5889 & 0.6197 & 0.5982 \\
\hline DYS444 & 654 & 0.5918 & 20 & 0.5738 & 0.5577 & 0.6690 \\
\hline DYS594 & 657 & 0.5374 & 21 & 0.3277 & 0.5480 & 0.5146 \\
\hline DYS556 & 655 & 0.5255 & 22 & 0.4263 & 0.5638 & 0.4486 \\
\hline DYS525 & 93 & 0.5157 & 23 & 0.3941 & 0.3871 & 0.6996 \\
\hline DYS632 & 94 & 0.5017 & 24 & 0.3701 & 0.2722 & 0.5141 \\
\hline DYS540 & 659 & 0.4409 & 25 & 0.3941 & 0.4432 & 0.5056 \\
\hline DYS641 & 94 & 0.1219 & 26 & 0.1287 & 0.2319 & 0.0000 \\
\hline DYS575 & 94 & 0.0213 & 27 & 0.0000 & 0.0625 & 0.0000 \\
\hline
\end{tabular}

The loci are listed according to their diversity values with the combined data set. Note that the total number of samples run for each locus varies, as does the ranking in each population group. future manuscript will address optimal loci in terms of haplotype resolution as well as construction of new large multiplexes to enable simultaneous amplification of the better performing loci.

Table 3 compares the gene diversity values for the individual loci in all the samples examined as well as the various U.S. population groups. Haplotype information across our U.S. samples for these 27 Y-STRs has not been included in the print version of this announcement due to space considerations but is available in electronic format at http://www.cstl.nist.gov/biotech/strbase/NISTpop.htm (data from Schoske et al. [7] on many of the same samples with 22 additional loci is also available on our website). Future reports are planned based on this data that will investigate the value of additional loci to those currently available in commercial STR kits.

This paper follows the guidelines for publication of population data requested by the journal [13].

\section{Acknowledgements}

This work was funded by the National Institute of Justice (NIJ) through an interagency agreement with the NIST Office of Law Enforcement Standards. Initial preparation of the population samples by Jan Redman and Richard Schoske is gratefully acknowledged. The Yfiler kits used to generate the DYS635 data were kindly provided by Applied Biosystems as part of a beta test and concordance study. Certain commercial equipment, instruments and materials are identified in order to specify experimental procedures as completely as possible. In no case does such identification imply a recommendation or endorsement by the National Institute of Standards and Technology nor does it imply that any of the materials, instruments or equipment identified are necessarily the best available for the purpose.

\section{References}

[1] J.M. Butler, R. Schoske, P.M. Vallone, J.W. Redman, M.C. Kline, Allele frequencies for 15 autosomal STR loci on U.S. Caucasian, African American, and Hispanic populations, J. Forensic Sci. 48 (2003) 908-911.

[2] S.A. Miller, D.D. Dykes, H.F. Polesky, A simple salting out procedure for extracting DNA from human nucleated cells, Nucleic Acids Res. 16 (1988) 1215.

[3] V.L. Singer, L.J. Jones, S.T. Yue, R.P. Haugland, Characterization of PicoGreen reagent and development of a fluorescence-based solution assay for double-stranded DNA quantitation, Anal. Biochem. 249 (1997) 228-238.

[4] P.M. Vallone, J.M. Butler, AutoDimer: a screening tool for primer-dimer and hairpin structures, Biotechniques 37 (2004) 226-231.

[5] P. Gill, C. Brenner, B. Brinkmann, B. Budowle, A. Carracedo, M.A. Jobling, P. de Knijff, M. Kayser, M. Krawczak, W.R. Mayr, N. Morling, B. Olaisen, V. Pascali, M. Prinz, L. Roewer, 
P.M. Schneider, A. Sajantila, C. Tyler-Smith, DNA commission of the International Society of Forensic Genetics: recommendations on forensic analysis using Y-chromosome STRs, Int. J. Leg. Med. 114 (2001) 305-309.

[6] M. Nei, Molecular Evolutionary Genetics, Columbia University Press, New York, 1987, pp. 176-179.

[7] R. Schoske, P.M. Vallone, M.C. Kline, J.W. Redman, J.M. Butler, High-throughput Y-STR typing of U.S. populations with 27 regions of the $\mathrm{Y}$ chromosome using two multiplex PCR assays, Forensic Sci. Int. 139 (2004) 107-121.

[8] A.J. Redd, A.B. Agellon, V.A. Kearney, V.A. Contreras, T. Karafet, H. Park, P. de Knijff, J.M. Butler, M.F. Hammer, Forensic value of 14 novel STRs on the human Y chromosome, Forensic Sci. Int. 130 (2002) 97-111.

[9] M. Kayser, R. Kittler, A. Ralf, M. Hedman, A.C. Lee, A. Mohyuddin, S.Q. Mehdi, Z. Rosser, M. Stoneking, M.A. Jobling, A. Sajantila, C. Tyler-Smith, A comprehensive survey of human Y-chromosomal microsatellites, Am. J. Hum. Genet. 74 (2004) 1183-1197.

[10] R. Iida, E. Tsubota, K. Sawazaki, M. Masuyama, T. Matsuki, T. Yasuda, K. Kishi, Characterization and haplotype analysis of the polymorphic Y-STRs DYS443, DYS444, and DYS445 in a Japanese population, Int. J. Leg. Med. 116 (2002) 191194.

[11] H.L. Dai, X.D. Wang, Y.B. Li, J. Wu, J. Zhang, H.J. Zhang, J.G. Dong, Y.P. Hou, Characterization and haplotype analysis of 10 novel Y-STR loci in Chinese Han population, Forensic Sci. Int. 145 (2004) 47-55.

[12] L. Gusmao, A. Gonzalez-Neira, C. Alves, M. Lareu, S. Costa, A. Amorim, A. Carracedo, Chimpanzee homologous of human Y specific STRs: a comparative study and a proposal for nomenclature, Forensic Sci. Int. 126 (2002) 129-136.

[13] P. Lincoln, A. Carracedo, Publication of population data of human polymorphisms, Forensic Sci. Int. 110 (2000) 3-5. 